\title{
Revisão da farmacoterapia de pacientes do Programa Componente Especializado da Assistência Farmacêutica em um município de Santa Catarina, Brasil
}

\author{
A pharmacotherapy review in patients attending \\ the Specialized Component For Pharmaceutical \\ Services in a Santa Catarina County, Brazil
}

Recebido em: 22/08/2016

Aceito em: $\quad 31 / 01 / 2017$
Graziela Modolon ALANO; Débora Mota Dal Bó LEGUIZAMONN;

Vanessa Mota VARGAS

Curso de Farmácia, Universidade do Sul de Santa Catarina - Unisul. Av. José Acácio Moreira, $n^{\circ} 787$, Bairro Dehon, Tubarão, SC.

CEP 88704-900, Brasil.E-mail: grazimod@gmail.com

\section{ABSTRACT}

The specialized component for pharmaceutical services (Componente Especializado da Assistência Farmacêutica $C E A F$ ) consists of a strategy to access high-cost treatments and new diseases that seeks to ensure full pharmaceutical treatment at the outpatient level. The aim of this study was to carry out a pharmacotherapy review in patients attending the CEAF program in a county at Santa Catarina, Brazil. This was a cross-sectional study through interviews conducted from August through September 2014. Twenty-two patients (59.9\% men; mean age 47.9 years; SD 15.2) were interviewed. Among them, $42.9 \%$ had completed high school, and 33.3\% had completed primary school. The average number of drugs per patient was 4.7 (SD 3.5), of which $30.1 \%$ were for the cardiovascular system and $26.2 \%$ for the nervous system. The most prevalent disease was schizophrenia (23.1\%). Among the participants, $54.6 \%$ had problems with the use of medications, such as dose schedule, interaction between medicines and interaction with food, and $90.9 \%$ had problems related to pharmaceutical therapy, such as medication storage and transport. These patients received oral instructions and review reports with written guidelines, schedules, and pictograms. The higher the number of drugs used, the more frequent the problems were. The participants reported that the pharmaceutical services helped them to take their medications correctly. This study highlights the importance of the specialized pharmaceutical services to achieve better therapeutic results, considering the high cost of treatments offered by the CEAF program.

Keywords: Pharmaceutical services; patient safety; Adherence to medication.

\section{RESUMO}

O CEAF (Componente Especializado da Assistência Farmacêutica) consiste em uma estratégia de acesso a tratamentos de alto custo e novas doenças que busca garantir a integralidade do tratamento medicamentoso em nível ambulatorial. O objetivo do estudo foi realizar a Revisão da Farmacoterapia em pacientes atendidos pelo programa CEAF em um município do Sul Catarinense. Tratou-se de um estudo transversal por meio de entrevistas realizadas entre agosto e setembro de 2014. Foram 22 pacientes com idade média de 47,9 (SD 15,2) anos, dos quais 59,9\% eram homens. Do total de entrevistados, $42,9 \%$ tinham o ensino médio completo e 33,3\%, nível fundamental. A média de medicamentos por paciente foi 4,7 (SD 3,5), sendo 30,1\% para o sistema cardiovascular e 26,2\% para o sistema nervoso. A doença mais prevalente foi a esquizofrenia $(23,1 \%)$. Foram identificados problemas relacionados com os medicamentos em $54,6 \%$ 
dos entrevistados, como intervalo de dose, interação medicamentosa e interação com alimentos. Foram identificadas necessidades relacionadas ao paciente em $90,9 \%$ dos entrevistados, na maioria orientações acerca dos produtos destinados à terapia farmacológica, como armazenamento e transporte. Para estes pacientes, foi entregue um relatório de revisão contendo o aprazamento, orientações escritas e pictogramas, acompanhados de orientações verbais. Problemas de uso dos medicamentos foram mais frequentes quanto maior o número de medicamentos utilizados. Os pacientes relataram que o serviço auxiliou na rotina de tomada de medicamentos. O estudo reforça a importância do serviço para o alcance do melhor resultado terapêutico considerando o alto custo dos tratamentos oferecidos pelo programa CEAF. Palavras-chave: Assistência Farmacêutica; segurança do paciente; adesão à medicação

\section{INTRODUÇÃO}

No Brasil, o Sistema Único de Saúde (SUS) foi criado para garantir a integralidade e igualdade nas ações e serviços do sistema público. De acordo com a Constituição Federal, devem ser garantidas, a todos os cidadãos, políticas que reduzam o risco de doenças, por meio de programas sociais que promovam a proteção e a recuperação da saúde do indivíduo (1).

A Assistência Farmacêutica é parte essencial da assistência à saúde, sendo o acesso aos medicamentos fundamental para o processo de atenção integral à saúde do indivíduo. Durante as últimas décadas, muitos avanços ocorreram no acesso aos medicamentos, dentre eles a adoção da Relação Nacional de Medicamentos Essenciais (RENAME). Como parte integrante da Política Nacional de Saúde, a RENAME deve garantir o acesso da população aos medicamentos mais seguros e eficazes, voltados ao tratamento das doenças mais prevalentes e disponibilizados em quantidade adequada à população $(1,2)$.

Os recursos para garantir a aquisição desses medicamentos estão regulamentados na Portaria no 204 de 29 de janeiro de 2007, a qual define os recursos para a compra desses produtos. A distribuição desses medicamentos está organizada em três esferas: medicamentos do componente básico, estratégico e excepcional, este último denominado atualmente como Componente Especializado da Assistência Farmacêutica (CEAF) (3).

O CEAF é uma forma de acesso aos tratamentos de alto custo, incluindo novas doenças, por meio do qual o Sistema Único de Saúde busca garantir a integralidade do tratamento medicamentoso em nível ambulatorial (4).

O Ministério da Saúde por meio do Programa de Medicamentos de Alto Custo, que inclui os medicamentos do componente especializado, disponibiliza aos pacientes os medicamentos de valor unitário muito elevado, bem como os medicamentos para tratamento de longa duração que, por se tratarem, muitas vezes, de doenças crônicas, tornam o tratamento dispendioso (5).

A terapia medicamentosa permite ao usuário tratar ou prevenir uma doença, atenuar os seus sintomas ou retardar a progressão da mesma e, dessa forma, o acesso aos medicamentos é algo fundamental. Por outro lado, a falta de informação sobre medicamentos compõe, muitas vezes, uma fonte de problemas devido ao potencial de causar Resultados Negativos associados à Medicação (RNM), os quais podem afetar a saúde durante o tratamento ou impedir o alcance dos objetivos terapêuticos $(6,7)$. Desse modo, tornam-se importantes estratégias de distribuição dos medicamentos que garantam não somente o acesso, mas também o repasse de informações ao paciente, necessárias para o uso correto, bem como a diminuição dos problemas relacionados a medicamentos (PRM), definido como aquela situação em que o processo de uso de medicamentos causa ou pode causar um RNM, como por exemplo, interação medicamentosa, reação adversa, entre outros $(7,8)$.

Diante do exposto, a revisão da farmacoterapia compreende um serviço farmacêutico de grande relevância, uma vez que neste processo, o profissional tem acesso às informações relacionadas ao paciente, sua rotina, tratamento e problemas de saúde; a partir destas informações, pode identificar e tentar solucionar os problemas encontrados na terapia farmacológica, como por exemplo, problemas de adesão e no intervalo de doses. Evidências comprovam que a revisão da farmacoterapia aumenta os resultados de saúde para os pacientes, podendo minimizar custos e riscos de hospitalização $(9,10)$.

A revisão da farmacoterapia é uma prática que busca organizar a medicação do paciente considerando as particularidades do medicamento em uso, como intervalo de dose e interação com alimento, a partir das orientações fornecidas pelo profissional prescritor e diante da rotina adotada pelo paciente (11).

Considerando a relevância do CEAF no acesso aos medicamentos de alto custo e a importância do fornecimento de orientações para a garantia de um tratamento 
seguro e eficaz, o presente estudo tem como finalidade realizar a revisão da farmacoterapia em um grupo de pacientes usuários de medicamentos do CEAF, buscando identificar possíveis problemas de uso da medicação, realizar as intervenções necessárias e avaliar a aceitação destas pelo paciente.

\section{MATERIAL E MÉTODOS}

Este projeto foi elaborado de acordo com as recomendações da Resolução n. ${ }^{\circ} 466$ de 12 de dezembro de 2012 do Conselho Nacional de Saúde, submetido e aprovado pelo Comitê de Ética em Pesquisa sob n. 737.842.

Foi realizado um estudo transversal por meio da aplicação de questionário estruturado a pacientes atendidos pelo CEAF. A população do estudo foi composta pelos pacientes que estavam utilizando o Programa do Componente Especializado da Assistência Farmacêutica de um município do sul do estado de Santa Catarina. Tal município possui aproximadamente 10.635 habitantes e, no momento do estudo, atendia 164 pacientes pelo programa CEAF $(12,13)$.

Para a seleção dos participantes foi adotado, como critério, a utilização dos medicamentos com maior preço, ofertados pelo Programa no município em estudo. Assim, foi estimado o gasto financeiro de cada medicamento do componente especializado nos meses de janeiro, fevereiro e março de 2014, com o intuito de mensurar os medicamentos mais onerosos financeiramente. Foram selecionados os primeiros cinco medicamentos de maior custo para o Estado para posterior seleção dos pacientes (Tabela 1).

Tabela 1: Lista dos cinco medicamentos mais onerosos no Programa Componente Especializado da Assistência Farmacêutica (CEAF) de um município de Santa Catarina, entre janeiro e março de 2014

\begin{tabular}{|l|l|l|l|}
\hline \multicolumn{1}{|c|}{ Medicamento } & \multicolumn{1}{|c|}{ Forma Farmacêutica } & Ação Farmacológica & Gasto Trimestral (R\$) \\
\hline dalimumabe 40mg & Solução injetável & Inibidor do fator de necrose tumoral & $132.016,00$ \\
\hline sevelamer 800mg & Comprimido & Removedor de fosfato & $111.300,00$ \\
\hline alfadornase 25mg & Solução para inalação & Proteína desoxirribonuclease & $22.245,00$ \\
\hline alfaepoetina 400UI & Solução injetável & Regulador da eritropoiese & $17.746,00$ \\
\hline olanzapina 10mg & Comprimido & Antipsicótico e anticonvulsivo & $13.549,00$ \\
\hline Total & & & $296.856,00$
\end{tabular}

Brasil, 2010 (1); SISMEDEX, 2014 (13)

Na primeira etapa do estudo foi solicitado ao farmacêutico da Unidade Central de Saúde do município um relatório contendo os usuários ativos dos medicamentos para que fosse freita a seleção da amostra. Estes pacientes foram, então, convidados a participar da pesquisa no mês de julho de 2014, por via telefônica. Havendo a concordância, foram orientados a apresentar todos os seus medicamentos em uso em um encontro previamente agendado.

Os encontros para a realização das entrevistas foram agendados para o mês de agosto de 2014, no mesmo período reservado à entrega dos medicamentos do CEAF, que compreende do $20^{\circ}$ ao $26^{\circ}$ dia de cada mês, por ordem de chegada dos pacientes. Foram excluídos da pesquisa os pacientes que não assinaram o Termo de Consentimento Livre e Esclarecido (TCLE), os menores de 18 anos e os pacientes com problemas de saúde agravados (alterações cognitivas, debilidade física). Durante as entrevistas, os pacientes foram questionados quanto sua rotina diária, incluindo os horários das refeições, de uso dos medicamentos, bem como os horários em que o paciente costuma acordar e ir dormir.

Após a coleta de dados, foi realizada a revisão da farmacoterapia com o intuito de analisar a rotina do paciente quanto ao uso dos medicamentos, enfatizando o estudo quanto ao modo de uso, posologia, interações com alimentos e outros medicamentos. Aos pacientes que, posteriormente à rotina analisada, foram identificados problemas no uso dos medicamentos elaborou-se o relatório de revisão, contendo uma proposta de rotina para o uso dos medicamentos, seguindo um modelo adaptado de Rigone e cols. (2015) (14). Os pacientes que necessitavam acompanhamento farmacoterapêutico 
foram encaminhados para o farmacêutico do município para dar continuidade ao serviço.

No primeiro encontro com o paciente foi ainda observada a existência de necessidades relacionadas ao paciente (NRP), as quais compreendem as deficiências de informações sobre o seu problema de saúde e o tratamento terapêutico, dentre outros encaminhamentos e orientações de saúde. Definida por Alano e cols. (2012), NRP é uma necessidade identificada pelo paciente ou pela equipe de saúde, relacionada com a falta ou a deficiência de informação que reflete na compreensão desta por parte do paciente. Desta forma, essas necessidades podem incluir orientações sobre o problema de saúde, a terapêutica e o sistema de saúde (15).

No mês de setembro foi então realizado um segundo encontro com os pacientes para a entrega do relatório de revisão. Após uma semana deste encontro, foi realizado um contato telefônico para investigar a facilidade de adoção das intervenções realizadas e a melhora da adesão ao tratamento após a oferta do serviço de revisão da farmacoterapia. A adesão ao tratamento foi mensurada segundo a percepção autorreferida do paciente, categorizada em: não tenho adesão (valor zero), quase nunca (valor 1), às vezes (valor 2) e, sempre tenho adesão (valor 3). Esse questionamento foi também realizado no primeiro encontro com o pesquisador. Além da adesão, foi mensurada a facilidade em seguir as recomendações das intervenções e/ou orientações fornecidas pelos pesquisadores, categorizando-as em: impossível, difícil, razoável e fácil.

Os medicamentos utilizados pelos pacientes entre- vistados foram classificados segundo a Anatomical Therapeutic Chemical (ATC) para padronizar a apresentação dos resultados, seguindo o modelo adotado por estudos na área da saúde (16).

Os dados foram analisados estatisticamente, utilizando-se o software Epi $\operatorname{Info}^{\circledR}$ versão 7.1.3. Foram calculadas a média, mediana e desvio padrão para as variáveis contínuas e proporções para as variáveis categóricas. Para análise da associação entre a variável de desfecho (necessidade de intervenção e/ou orientação do farmacêutico) e as variáveis de exposição (perfil dos entrevistados) foi adotado o teste do Qui-quadrado exato de Fisher. O nível de significância pré-estabelecido foi de $95 \%$ ( $p$ menor que 0,05 ).

\section{RESULTADOS E DISCUSSÃO}

Com base nos medicamentos previamente selecionados pelo critério de alto custo, foram incluídos na amostra 28 pacientes em uso dessas medicações no mês de julho. Desses, 22 pacientes se enquadraram no critério de inclusão e participaram das entrevistas; dos demais, um não pode ser incluído por ter interrompido o uso do medicamento; um por não aceitar a própria doença, portanto, não realizando o tratamento; um por não aceitar participar da entrevista; um por estar acamado e dois por não possuírem capacidade cognitiva para participar da entrevista devido ao seu problema de saúde (esquizofrenia). A Tabela 2 apresenta a relação dos medicamentos, as doenças de origem e o número de perdas de pacientes da amostra.

Tabela 2: Medicamentos, doenças e número de pacientes entrevistados no Programa Componente Especializado da Assistência Farmacêutica (CEAF) de um município de Santa Catarina, entre janeiro e março de 2014

\begin{tabular}{|l|l|c|c|c|}
\hline \multicolumn{1}{|c|}{ Medicamento } & \multicolumn{1}{c|}{ Doença } & $\begin{array}{c}\text { Número de } \\
\text { Pacientes }\end{array}$ & $\begin{array}{c}\text { Pacientes } \\
\text { Entrevistados }\end{array}$ \\
\hline adalimumabe & Doença de Crohn/ Artrite Reumatóide & 4 & 1 & 3 \\
\hline sevelamer & Fibrose Cística & 2 & 0 & 2 \\
\hline alfadornase & IRC* & 1 & 0 & 1 \\
\hline alfaepoetina & IRC* & 6 & 0 & 6 \\
\hline olanzapina & Esquizofrenia & 15 & 5 & 10 \\
\hline Total & 28 & 6 & 22 & \\
\hline
\end{tabular}

*Insuficiência Renal Crônica 
Devido ao não comparecimento à Unidade Central de Saúde na primeira etapa da pesquisa e para evitar maior redução do tamanho da amostra, foi decidido, então, realizar, em alguns casos, visitas domiciliares.

Foram realizadas as revisões da farmacoterapia para os 22 pacientes entrevistados. Esses possuíam idade média de 47,9 anos (SD 15,7) sendo a idade mínima 19,5 anos e a idade máxima 75,1 anos, dos quais $59,9 \%$ eram homens.

A Tabela 3 apresenta as principais características sociodemográficas dos entrevistados. Quanto à escolaridade, $42,9 \%$ tinham o ensino médio completo e 33,3\% apresentaram nível de escolaridade primário.

A Tabela 4 mostra os problemas de saúde relatados pelos entrevistados, sendo a Esquizofrenia e a Insuficiência Renal Crônica (ICR) os mais prevalentes, afetando $23,1 \%$ e $20,5 \%$ dos pacientes, respectivamente

$\mathrm{O}$ número de medicamentos utilizados pelos entrevistados foi de 54 especialidades. Considerando que alguns medicamentos eram utilizados por mais de um paciente foi observado um total de 103, o que resultou em uma média de 4,7 (SD 3,5) medicamentos por paciente e uma variação de 1 a 11 medicamentos por paciente

Tabela 3: Distribuição das variáveis sociodemográficas dos pacientes entrevistados no Programa Componente Especializado da Assistência Farmacêutica (CEAF) de um município de Santa Catarina, entre janeiro e março de 2014

\begin{tabular}{|c|c|c|}
\hline Variáveis & \multirow{2}{*}{ n (22) } & \multirow{2}{*}{$\%$} \\
\hline Idade (anos) & & \\
\hline $18-39$ & 6 & 27,3 \\
\hline $40-59$ & 10 & 45,4 \\
\hline $60-80$ & 6 & 27,3 \\
\hline \multicolumn{3}{|c|}{ Nivel de Escolaridade } \\
\hline Não estudou & 2 & 9,5 \\
\hline Ensino primário & 10 & 33,3 \\
\hline Ensino fundamental & 2 & 9,5 \\
\hline Ensino médio & 9 & 42,9 \\
\hline Superior & 1 & 4,8 \\
\hline
\end{tabular}

.De acordo com a classificação ATC (Anatomical Therapeutic Chemical), os fármacos mais utilizados foram os relacionados ao sistema cardiovascular $(30,1 \%) \mathrm{e}$ sistema nervoso $26,2 \%$, sendo o anlodipino e a olanzapina os maiores representantes destas classes, respectivamente. Segundo informações dos entrevistados, todos os medicamentos foram prescritos pelo médico.
Tabela 4: Agravos de saúde relatados pelos pacientes entrevistados no Programa Componente Especializado da Assistência Farmacêutica (CEAF) de um município de Santa Catarina, entre janeiro e março de 2014

\begin{tabular}{|l|l|c|}
\multicolumn{1}{c}{ Agravos de Saúde } & n & $\%$ \\
\hline Esquizofrenia* $^{*}$ & 9 & 23,1 \\
\hline Insuficiência Renal Crônica* & 8 & 20,5 \\
\hline Hipertensão & 6 & 15,3 \\
\hline Diabetes & 4 & 10,2 \\
\hline Depressão & 4 & 10,2 \\
\hline Artrite Reumatoide* & 2 & 5,1 \\
\hline Trombose & 1 & 2,6 \\
\hline Câncer & 1 & 2,6 \\
\hline Dislipidemia & 1 & 2,6 \\
\hline Síndrome do Pânico & 1 & 2,6 \\
\hline Fibrose Cística* & 1 & 2,6 \\
\hline Doença de Crohn* & 1 & 2,6 \\
\hline
\end{tabular}

Doença em que o tratamento medicamentoso pertence ao CEAF

Após a análise dos dados foram identificados problemas relacionados com os medicamentos em 54,6\% dos entrevistados e, em $90,9 \%$ dos casos. Foram identificadas necessidades relacionadas ao paciente; em sua maioria, necessidades de orientações acerca da terapia farmacológica (Tabela 5).

Dentre os pacientes, $40,9 \%$ foram encaminhados para acompanhamento com o farmacêutico do município devido à necessidade de monitoramento do tratamento e/ ou contato com o prescritor para solucionar ou prevenir os problemas identificados. Nesses casos, o serviço de acompanhamento farmacoterapêutico se fez necessário.

A rotina de tomada dos medicamentos dos pacientes foi analisada para investigar se houve um aumento do número de tomadas diárias após a realização das intervenções pelos pesquisadores, sendo que a média anterior ao serviço de revisão da farmacoterapia foi de 2,9 (SD 1,6; 1 a 6 tomadas diárias) passando para 3,0 (SD 1,7; 1 a 7 tomadas diárias). Todos os pacientes receberam o relatório de revisão e, nos casos, onde não houve necessidade de intervenção, a rotina de uso da medicação foi transcrita conforme o relato do paciente.

Quanto à adesão ao tratamento, 77,3\% declararam seguir "sempre" as recomendações médicas quanto ao uso dos medicamentos. No segundo contato, $100 \%$ dos pacientes que tiveram retorno (20) declararam seguir "sempre" as recomendações médicas, bem como as 
orientações fornecidas pelos pesquisadores, demonstrando uma aceitação total das intervenções e orientações. Neste mesmo contato, os pacientes responderam quanto à facilidade de seguir as recomendações realizadas, feitas por meio das intervenções e/ou orientações, onde 95\% declararam como sendo de fácil entendimento e execução, apenas um dos pacientes declarou ser razoável.

Por meio de análises de associação (Qui-quadrado exato de Fisher), foi constatado que somente o número de medicamentos (três ou mais) utilizados pelo paciente foi associado ao fato de possuir necessidade de intervenção farmacêutica na rotina de uso dos medicamentos $(p=0,0062)$. Não foi encontrada relação significativa entre o gênero dos entrevistados e haver necessidade de intervenção $(p=0,4149)$ ou necessidade de orientação $(p=0,4935)$, bem como a idade e a necessidade de intervenção $(p=0,646)$ ou necessidade de orientação $(p=0,481)$; e, ainda, entre o número de problemas de saúde e necessidade de intervenção $(p=1,0)$. Da mesma forma, não houve relação significativa entre necessidade de encaminhamento ao farmacêutico para acompanhamento e o gênero do entrevistado $(p=1,0)$. Cabe ressaltar que o tamanho reduzido da amostra dificultou a análise estatística dos dados.
Dentre as doenças prevalentes, neste estudo a esquizofrenia foi a mais frequente e, portanto, o medicamento com maior utilização foi a olanzapina, bem como no estudo de Blatt (2005), que encontrou o mesmo medicamento como sendo um dos mais solicitados por meio do CEAF (17). A população em geral está sujeita a ter problemas com o uso de seus medicamentos e, dessa forma, os pacientes que possuem esquizofrenia acabam, muitas vezes, mais expostos devido ao estado de sua condição mental, que pode dificultar o entendimento do modo correto de usá-los, necessitando em muitos casos, do auxílio de um cuidador. No que diz respeito à promoção da saúde e, principalmente, saúde mental, apenas o fornecimento do medicamento não é capaz de garantir o bem-estar do paciente, sendo necessárias ações de educação em saúde e cuidados farmacêuticos envolvendo tanto o paciente quanto seu cuidador e família, com o intuito de minimizar os riscos do tratamento e gerar melhores resultados terapêuticos. Nesses casos, o profissional precisa não somente de um conhecimento teórico bem sedimentado, mas também a habilidade de lidar com a subjetividade do paciente (18).

Tabela 5: Problemas relacionados aos medicamentos e necessidades relacionadas ao paciente entrevistados no Programa Componente Especializado da Assistência Farmacêutica (CEAF) de um município de Santa Catarina, entre janeiro e março de 2014

\begin{tabular}{|c|c|c|}
\hline Variáveis & n (16) & $\%$ \\
\hline $\begin{array}{l}\text { Problema relacionado ao medicamento } \\
\text { Intervalo de dose } \\
\text { Interação medicamento-alimento } \\
\text { Interação medicamento-medicamento } \\
\text { Não adesão ao tratamento } \\
\text { Efeito adverso }\end{array}$ & $\begin{array}{l}8 \\
3 \\
1 \\
3 \\
1\end{array}$ & $\begin{array}{r}50,0 \\
18,8 \\
6,2 \\
18,8 \\
6,2\end{array}$ \\
\hline $\begin{array}{l}\text { Necessidade relacionada ao paciente } \\
\text { Orientação sobre transporte e armazenamento do medicamento } \\
\text { Mudança de hábitos de vida (fumo e álcool) } \\
\text { Monitoramento da pressão arterial e dosagem de lítio }\end{array}$ & $\begin{array}{c}n(23) \\
11 \\
\\
8 \\
4\end{array}$ & $\begin{array}{c}\% \\
47,8 \\
\\
34,8 \\
17,4\end{array}$ \\
\hline
\end{tabular}

Dentre os problemas com a administração dos medicamentos identificados neste estudo, o intervalo de dose foi o mais prevalente entre os pacientes que necessitaram de intervenção. Um paciente, por exemplo, relatou não se sentir bem após a tomada da dose de $10 \mathrm{mg}$ da olanzapina pela manhã, além da dose de $5 \mathrm{mg}$ à noite. De acordo com a literatura, a meia-vida da olanzapina é aproximadamente 33 horas (21 a 54 horas) e, portanto, sua administração deve ser em dose única diária com dose máxima de até $20 \mathrm{mg}(19,20)$. Além do tempo de meia-vida, outros fatores podem estar relacionados aos problemas de intervalo de dose, como a falta de entendimento pelos pacientes de como utilizar os medicamentos, ou mesmo, o comprometimento do paciente em relação ao seu tratamento por meio da adesão terapêutica, estando estes, entre as principais causas de problemas no tratamento medicamentoso (21).

Galato e cols. (2008) destacam que a informação sobre o horário correto da medicação deve ser claramente informada ao paciente, principalmente, no início do tratamento e que, de preferência, deverá sempre que possível ser negociado com o paciente o melhor horário 
para assim facilitar a adesão, contribuindo para o alcance do resultado esperado (22). Neste estudo, nos casos onde foi necessário ajuste de horário, foi priorizada a negociação do melhor horário para o paciente, diante da característica do medicamento.

Todos os pacientes receberam o relatório de revisão e para aqueles onde não houve necessidade de intervenção, a rotina de uso da medicação foi transcrita conforme o relato do paciente. Este relatório de revisão entregue aos pacientes constituiu em um documento que organizava os medicamentos a serem utilizados em: quantidade a ser ingerida, aprazamento (horários de administração) e os cuidados com a alimentação. Além disso, o relatório continha também a descrição das intervenções e outras informações necessárias ao paciente, como necessidade de monitoramento por exames laboratoriais e controle da pressão arterial. Sempre que possível, foram utilizados pictogramas para facilitar o entendimento e buscou-se interferir o mínimo possível na rotina pré-estabelecida pelo paciente.

$\mathrm{O}$ aprazamento realizado pode, em alguns casos, aumentar a complexidade do tratamento, de acordo com os resultados do trabalho realizado em uma população de hipertensos (21). Neste estudo, se observou um pequeno aumento do número médio de tomadas diárias de medicamentos.

Ainda em relação ao medicamento olanzapina, houve o relato de um efeito adverso onde o paciente relacionou o ganho de peso de aproximadamente $20 \mathrm{~kg}$ após o início de uso deste medicamento. Segundo a literatura, o ganho de peso está entre os efeitos adversos mais frequentes em relação ao uso da olanzapina $(19,20)$. Nessa situação, bem como no caso do problema no intervalo de doses, descrito anteriormente, os pacientes foram informados dos dados da literatura e encaminhados ao farmacêutico da Unidade Central de Saúde para a avaliação da possibilidade de intervenção junto ao prescritor.

A interação entre o medicamento e o alimento foi identificada em três dos pacientes entrevistados. Nestes, a interação pode resultar em alteração na absorção do medicamento como, por exemplo, no caso do omeprazol que deve ser administrado em jejum. Nesses casos, a intervenção consistiu em distanciar o horário de administração do medicamento e a alimentação. Magedanz e cols. (2009) citaram em sua pesquisa realizada no Hospital de Clínicas de Porto Alegre, a alta incidência de prescrições contendo medicamentos possíveis de interagir com os alimentos, ressaltando a importância de orientações relacionadas à correta administração dos medicamentos (23).
A interação medicamentosa encontrada nesse estudo ocorreu entre a atorvastatina e o diltiazem. Segundo a literatura, o diltiazem pode aumentar os níveis sanguíneos da atorvastatina, podendo assim, causar efeitos secundários como danos no fígado e rabdomiólise. Esta última condição, embora rara, é bastante grave, sendo necessário o monitoramento do paciente (24,25). Franco e cols. (2013) e Lewin e cols. (2002) destacaram a relevância dessa interação, sendo necessário avaliar custo e benefício do tratamento ao paciente, embora nenhum relato de reação adversa tenha sido associado a essa interação $(26,27)$. O paciente foi encaminhado para acompanhamento junto do farmacêutico da Unidade Central de Saúde.

Foram identificadas necessidades relacionadas ao paciente que, em sua maioria, compreenderam a necessidade de orientações acerca da terapia farmacológica, assim como observado no estudo de Alano e cols. (2012) onde $30,4 \%$ foram informações sobre a terapia farmacológica (15). A necessidade de monitoramento do paciente foi observada nos dois estudos, sendo $17,4 \%$ e $19,2 \%$, respectivamente. É importante destacar que ambos os estudos mostraram um elevado número de pacientes apresentando dificuldade com as informações em saúde, seja por entendimento ou mesmo a falta de acesso a estas, o que pode influenciar no alcance dos resultados terapêuticos (15).

As intervenções propostas foram aceitas pela totalidade dos pacientes, embora o valor possa estar superestimado visto que o questionamento realizado pelo pesquisador foi por contato telefônico. Apesar disso, o resultado difere de dois trabalhos realizados em farmácias comunitárias, onde no primeiro houve $53 \%$ de aceite das intervenções envolvendo o médico e/ou paciente, e no segundo estudo, somente $47 \%$ de aceite para as intervenções envolvendo o médico; sendo que outro trabalho realizado em uma policlínica da Universidade atingiu 79\% de aceitação envolvendo intervenções com médico e/ou paciente $(15,27,28)$. A situação pode demonstrar que intervenções que necessitam do auxílio do prescritor apresentam maior dificuldade de resolução, seja por apresentarem-se mais complexa ou mesmo, por ainda enfrentarem outras barreiras no contato profissional. Outros estudos podem melhor investigar esses obstáculos. Como já mencionado, no presente estudo, as intervenções que necessitavam de auxílio médico foram encaminhadas para que o farmacêutico da Unidade de Saúde pudesse realizar o trabalho com a equipe de saúde.

Quanto à facilidade de seguir as recomendações realizadas no processo de revisão, apenas um dos pacientes declarou ser "razoável" a facilidade de segui- 
-las. Esse mesmo paciente relatou na primeira entrevista não aderir às medicações durante os finais de semana, onde, então, foi feita a orientação para conscientizar a importância da adesão ao tratamento para se alcançar os efeitos terapêuticos, bem como dos riscos advindos da não adesão. Todos os pacientes do estudo relataram, ainda, que o serviço fornecido de revisão da farmacoterapia contribuiu para auxiliar na sua rotina de tomada de medicamentos, pois obtiveram um maior entendimento sobre sua doença e sobre seus medicamentos.

Durante as entrevistas foi observado que alguns pacientes não adotavam cuidados necessários quanto ao armazenamento e o transporte dos medicamentos alfaepoetina e adalimumabe. Devido a isso, foi considerado que esses pacientes necessitavam se conscientizar quanto à importância do correto manuseio desses medicamentos. Neste caso, ambos os medicamentos necessitam de refrigeração, devendo ser conservados entre $2{ }^{\circ} \mathrm{C}$ e $8{ }^{\circ} \mathrm{C}$, transportados com cautela (caixa de isopor, por exemplo) para manter a temperatura adequada e evitar agitação do frasco $(19,24,28,29)$. Souza e cols. (2008) também citaram em seu estudo a necessidade de orientação aos pacientes sobre armazenamento de medicamentos de administração parenteral, assim como a importância da refrigeração para inibir a multiplicação microbiana e garantir a efetividade dos medicamentos que exigem baixa temperatura (30).

Do mesmo modo, foram necessárias orientações sobre hábitos de vida, em especial ao consumo de álcool e o fumo. Segundo a literatura, o uso do cigarro aumenta $40 \%$ do clearance da olanzapina no organismo, sendo que o uso do álcool durante o tratamento potencializa o efeito do medicamento no Sistema Nervoso Central, podendo causar sedação do paciente (20). Em relação ao fumo, Mendes (2012) em seu estudo sobre a bioequivalência entre duas formulações de olanzapina, encontrou que o clearance desse medicamento foi maior entre homens fumantes (31). Nesse estudo, dois pacientes que utilizavam olanzapina declararam ser fumantes e foram, então, informados dos riscos.

Foi possível constatar uma associação entre o uso de um número maior de medicamentos (três ou mais) e a necessidade de intervenção farmacêutica. $\mathrm{O}$ risco de problemas relacionados ao tratamento medicamentoso aumenta de três a quatro vezes em pacientes submetidos à polifarmacia, segundo Secoli (2010). Esse mesmo autor encontrou em seu estudo que, a incidência de problemas no uso da medicação como consequência da polifarmácia em idosos foi $15 \%$ quando utilizado um medicamento, porém elevando-se para $35 \%$ quando o número foi igual ou superior a quatro (32).

O problema da não adesão ao tratamento pelo paciente foi constatado nesse estudo, onde três deles relataram ter dificuldade de manter a terapia medicamentosa diária ou de administrar os medicamentos no horário correto. Estudos realizados em diversos países mostram que $50 \%$ a $60 \%$ dos pacientes que recebem uma prescrição médica não cumprem o tratamento estabelecido ou o interrompem quando se sentem melhores, sendo necessário sempre que possível um reforço verbal ou escrito das informações, assim como foi realizado no presente estudo $(21,22)$.

Após a prestação do serviço de revisão da farmacoterapia, os pacientes foram novamente questionados quanto à adesão ao tratamento e todos se declararam aderentes ao tratamento. Entretanto, a adesão por autopercepção é normalmente superestimada pelos pacientes, tanto por uma percepção equivocada do conceito de adesão, quanto por receio de recriminação, além da interferência do viés de memória (33).

Todos os problemas verificados na descrição da rotina do paciente e as intervenções realizadas no processo de revisão da farmacoterapia de cada paciente foram registrados também em um relatório geral do estudo, o qual foi entregue ao farmacêutico da Unidade Central de Saúde. Neste mesmo relatório, foram registrados os casos de pacientes onde a intervenção somente poderia ser realizada mediante contato com o prescritor, destacando a necessidade de acompanhamento farmacoterapêutico nestas situações.

Neste estudo muitas variáveis não demonstraram relevância estatística, provavelmente, devido ao número reduzido da amostra. Trabalhos desta natureza, que incluem estudos de revisão da farmacoterapia e acompanhamento farmacoterapêutico, frequentemente encontram problemas desta ordem, fato que se confirma na revisão sistemática realizada por Nkansah e cols. (2010), na qual foram analisados os resultados de intervenções farmacêuticas de estudos publicados até 2011, mostrando que em estudos com menos de 150 pacientes é difícil encontrar relevância estatística (34). Além disso, o estudo envolveu pacientes com problemas de saúde, por vezes, com agravamentos ou situações complicadas que contribuíram para a redução da amostra. Apesar disso, o fato dos participantes relatarem que o serviço de revisão da farmacoterapia possibilitou o melhor entendimento de sua condição de saúde, bem como de seu tratamento, reforça a importância deste trabalho e destaca a necessi- 
dade de se realizar outros estudos mais prolongados que mostrem a influência deste serviço sobre a qualidade de vida do paciente ou desfecho clínico.

\section{CONCLUSÃO}

$\mathrm{Na}$ análise da rotina de uso dos medicamentos utilizados pelos pacientes atendidos pelo CEAF forma encontrados problemas relacionados aos medicamentos e necessidades de orientações acerca da terapia farma-

\section{REFERÊNCIAS}

1. BRASIL. Ministério da Saúde. Secretaria de Ciência, Tecnologia e Insumos Estratégicos Departamento de Assistência Farmacêutica e Insumos Estratégicos. Da excepcionalidade às linhas de cuidado: o Componente Especializado da Assistência Farmacêutica. Brasília: Ministério da Saúde, 2010. 262 p.

2. BRASIL. Ministério da Saúde. Formulário terapêutico nacional 2010: Rename 2010/Ministério da Saúde, Secretaria de Ciência, Tecnologia e Insumos Estratégicos, Departamento de Assistência Farmacêutica e Insumos Estratégicos. 2a ed. Brasília: Ministério da Saúde, 2010. $1135 \mathrm{p}$.

3. BRASIL. Ministério da Saúde. Portaria $n^{\circ} 204$, de 29 de janeiro de 2007. Regulamenta o financiamento e a transferência dos recursos federais para as ações e os serviços de saúde, na forma de blocos de financiamento, com o respectivo monitoramento e controle. Diário Oficial da União, Brasília (DF) 2007, 31 de janeiro de 2007; 22, Seção 1. p. 45-50.

4. Vieira FS. Assistência farmacêutica no sistema público de saúde no Brasil. Rev Panam Salud Pública, 2010; 27(2):149-156.

5. Carias CM, Vieira FS, Giordano CV, Zucchi P. Medicamentos de dispensação excepcional: histórico e gastos do Ministério da Saúde do Brasil. Rev. Saúde Pública. 2011; 45(2): 233-240. DOI: 10.1590/S003489102011000200001

6. Lima-Dellamora EC, Caetano R, Osorio-de-Castro CGS. Dispensação de medicamentos do componente especializado em polos no Estado do Rio de Janeiro. Ciênc. Saúde Colet. 2012; 17(9):2387-96. DOI: 10.1590/S141381232012000900019

7. Comitê del Consenso. Tercer Consenso de Granada sobre Problemas Relacionados con Medicamentos (PRM) y Resultados Negativos Asociados a la Medicación (RNM). Ars Pharm. Granada, 2007; 48(1): 5-17.

8. Vieira FS. Possibilidades de contribuição do farmacêutico para a promoção da saúde. Ciênc. Saúde Colet. 2007; 12(1):213-20. DOI: 10.1590/S141381232007000100024 . cológica, prevalecendo problemas no intervalo de dose e orientações sobre o correto armazenamento e transporte. As intervenções e orientações repassadas tiveram aceitação pelos pacientes e foram consideradas de fácil entendimento pela maioria destes. Houve um aumento da adesão ao tratamento medicamentoso de acordo com a percepção autorreferida e muitos consideraram que o serviço de revisão da farmacoterapia possibilitou o melhor entendimento sobre seu problema de saúde e seus medicamentos.

9. Rozenfeld S. Prevalência, fatores associados e mau uso de medicamentos entre os idosos: uma revisão. Cad. Saúde Pública. 2013; 19(3):717-24. DOI: 10.1590/S0102311X2003000300004

10. Freeman C, Cottrell WN, Kyle G, Williams I, Nissen L. Integrating a Pharmacist into the General Practice Environment: Opinions of Pharmacist's, General Practitioner's, Health Care Consumer's, and Practice Manager's. BMC Health Services Research. 2012; 12:229. DOI: 10.1186/1472-6963-12-229

11. Holland R, Desborough J, Goodyer L, Hall S, Wright D, Loke YK. Does pharmacist-led medication review help to reduce hospital admissions and deaths in older people? A systematic review and meta-analysis. Br J Clin Pharmacol. 2007; 65(3):303-16.

12. IBGE. Instituto Brasileiro de Geografia e Estatística. Censo Demográfico 2010. Disponível em: http://www. ibge.gov.br/home/estatistica/populacao/censo2010/default.shtm

13. SISMEDEX. Sistema informatizado de gerenciamento e acompanhamento dos medicamentos excepcionais. Disponível em: http:/www.sismedex.saude.sc.gov.br:8080/ cmde uf/entrada.do?action= concluirProcesso\&action=concluirProcesso

14. Rigoni CC, Brito ES, Alano GM, Galato D. Pharmacotherapy review: a proposal to improve medication adherence among hypertensive patients. Braz J Pharm Sci. 2015; 5(4):763-773. DOI: 10.1590/S198482502015000400002

15. Alano GM, Corrêa TS, Galato D. Indicadores do Serviço de Atenção Farmacêutica (SAF) da Universidade do Sul de Santa Catarina. Ciênc Saúde Colet. 2012; 17(3):75764. DOI: $10.1590 / \mathrm{S} 1413-81232012000300023$

16. Collaborating Centre For Drugs Statistics Methodology. Anatomical Therapeutic Chemical Classification (ATC code) 2014. Disponível em: http://www.whocc.no/atc ddd_publications/guidelines/

17. Blatt CR. Avaliação da Assistência Farmacêutica do Programa de Medicamentos Excepcionais do Estado de Santa Catarina no ano de 2002 a 2004. [Dissertação]. 
Florianópolis: Universidade Federal de Santa Catarina UFSC; 2005.

18 Gomes EF. Importância da assistência e da atenção farmacêutica aplicada a pacientes com transtornos mentais. [Monografia]. Vitória: Faculdade Católica Salesiana Do Espírito Santo- FCSES; 2013. Disponível em: http:// www.catolica-es.edu.br/fotos/files/IMPORTANCIA $\% 20$ DA $\% 20$ ASSISTENCIA $\% 20 \mathrm{E} \% 20 \mathrm{DA} \% 20 \mathrm{ATEN}-$ CAO\%20FARMACEUTICA.pdf

19. Lacy CF, Lora L, Armstrong LL, Lance LL, Goldman MP (Ed). Drug Information Handbook: A Comprehensive Resource for All Clinicians and Healthcare Professionals. $22^{\mathrm{a}}$ ed. Hudson, Ohio:Lexi-Comp. 2013.

20. Frota LH , Brasil MAA, Alves JMF, Silva Filho JF. Olanzapine-Prelimminary Official Guidelines For Brazilian Mental Health Services. Critical Appraisal and Suggestions. J Bras Psiquiatr. 2001; 50(1/2):23-34.

21. Divaldo PLJ, Amaral RT, Veiga EV, Cárnio EC, Nogueira MS, Pelá IR. A farmacoterapia no idoso: revisão sobre a abordagem multiprofissional no controle da hipertensão arterial sistêmica. Rev Lat-am Enferm. 2006; 14(3):435-41.

22. Galato D, Alano GM, Trauthman SC, Vieira AC. A dispensação de medicamentos: uma reflexão sobre o processo para prevenção, identificação e resolução de problemas relacionados à farmacoterapia. Rev. Bras. Cienc. Farm. 2008; 44(3):465-475. DOI: 10.1590/S151693322008000300017

23. Magedanz L, Jacoby T, Silva D, Santos L, Martinbiancho J, Zuckermann J. Implementação de um programa para evitar possíveis interações fármaco-alimento em pacientes adultos internados em unidades clínicas e cirúrgicas de um hospital universitário. Rev HCPA. 2009; 29(1):29-32.

24. USP DI. United States Pharmacopeial Dispensing Information. Drug information for the health care professional. 25th ed. Vol. I. Rockville: The United States Pharmacopoeial Convention; 2005.

25. Fonseca FAH. Farmacocinética das estatinas. Arq. Bras. Cardiol. 2005; 85(5):09-14. DOI: 10.1590/S0066782X2005002400003
26. Franco D, Henao Y, Monsalve M, Gutiérrez F, Hincapie J, Amariles P. Interacciones medicamentosas de agentes hipolipemiantes: aproximación para establecer y valorar surelevancia clínica: revisión estructurada. Farm Hosp. 2013; 37(6):539-57.

27. Lewin JJ, Nappi JM, Taylor MH. Rhabdomyolysis with concurrent atorvastatin and diltiazem. Ann Pharmacother. 2002; 36(10):1546-9.

28. Dodson SE, Ruisinger JF, Roward PA, Hare SE, Barnes BJ. Community pharmacy-based medication therapy management services: financial impact for patients. Pharm Pract. 2012; 10(3):119-124.

29. Doucette WR, Mcdonough RP, Klepser D, Mccarthy R. Comprehensive medication therapy management: identifying and resolving drug-related issues in a community pharmacy. Clin Ther. 2005; 27(7):1104-11.

30. Souza MCP, Goulart MA, Rosado V, Reis AMM. Utilização de medicamentos parenterais em frascos-ampola em uma unidade pediátrica de um hospital universitário. Rev. Esc. Enferm. USP. 2008; 42(4):715-22.

31. Mendes, S. Comparação da bioequivalência entre duas formulações de olanzapina $2,5 \mathrm{mg}$ comprimidos em voluntários sadios após a administração de dose única. RBM Rev Bras Med. 2012; 69(7):21-25.

32. Secoli SR. Polifarmácia: interações e reações adversas no uso de medicamentos por idosos. Rev Bras Enferm. 2010; 63(1):136-140. DOI: 10.1590/S003471672010000100023

33. Silva T, Schenkel EP, Mengue SS. Nível de informação a respeito de medicamentos prescritos a pacientes ambulatoriais de hospital universitário. Cad. Saúde Pública. 2000; 16(2):449-55. DOI: 10.1590/ S0102-311X2000000200015

34. Nkansah N, Mostovetsky O, Yu C, Chheng T, Beney J, Bond CM, Bero L. Effect of outpatient pharmacists' non-dispensing roles on patient outcomes and prescribing patterns. Cochrane Data base Syst Rev. 2010; 7(7):120134. DOI: 10.1002/14651858.CD000336.pub2. 